\title{
Salvianolic Acid-A Induces Apoptosis, Mitochondrial Membrane Potential Loss and DNA Damage in Small Cell Lung Cancer Cell Lines
}

\author{
Le-Qiang Wang ${ }^{1}$, De-Feng Hua ${ }^{2}$, Xiao-Meng Li ${ }^{1}$, Juan $\mathrm{Li}^{1}$ and Yun Guan ${ }^{1 *}$ \\ ${ }^{1}$ Department of Respiratory, Weifang People's Hospital, ${ }^{2}$ Department of Neurology, Brain Hospital of Weifang People's \\ Hospital, Weifang 261599, China
}

*For correspondence: Email: guanyun885@gmail.com, guanyun885@126.com; Tel/Fax: 0086-536-8192599

\begin{abstract}
Purpose: To examine the anticancer effect of salvianolic acid-A against human small cell lung cancer (SCLC) cells (H-69).

Methods: In vitro antiproliferative effect of salvianolic acid against SCLC cell lines was evaluated by 3(4,5-dimethylthiazol-2-yl)-2,5-diphenyltetrazolium bromide (MTT) assay. Apoptosis induction by salvianolic acid-A in these cells was measured by videomicroscopy along with fluorescence microscopy using Hoechst 33258 staining. The effect of the compound on mitochondrial membrane potential loss was detected by flow cytometry using rhodamine-123 as fluorescent probe. Gel electrophoresis was used to analyze DNA fragmentation after salvianolic acid treatment.

Results: The results revealed that salvianolic acid- $A$ induces dose-dependent as well as timedependent growth inhibitory effects against SCLC cancer cells. Videomicroscopy analysis revealed that $S C L C$ cells became rounded (dead) and refringent after drug treatment which increased with increase in salvianolic acid-A dose. Fluorescence microscopy demonstrated that salvianolic acid-A induced dosedependent chromatin condensation in SCLC cells which is characteristic of apoptosis. DNA fragmentation was induced by various doses of the compound again hinting at apoptosis. Flow cytometry analysis using rhodamine-123 showed that salvianolic acid-A also caused substantial loss of mitochondrial membrane potential.

Conclusion: The results suggest that salvianolic acid-A is a potential anticancer and apoptotic agent against drug-resistant small cell lung cancer.
\end{abstract}

Keywords: Lung cancer, Apoptosis, Salvianolic acid-A, Flow cytometry, Anticancer activity, Videomicroscopy, Fluorescence microscopy, Mitochondrial membrane potential, Rhodamine-123

\begin{abstract}
Tropical Journal of Pharmaceutical Research is indexed by Science Citation Index (SciSearch), Scopus, International Pharmaceutical Abstract, Chemical Abstracts, Embase, Index Copernicus, EBSCO, African Index Medicus, JournalSeek, Journal Citation Reports/Science Edition, Directory of Open Access Journals (DOAJ), African Journal Online, Bioline International, Open-J-Gate and Pharmacy Abstracts
\end{abstract}

\section{INTRODUCTION}

Lung cancer is believed to be one of the most lethal carcinomas in the world $[1,2]$. In China, about 320,000 people died in 2005 because of lung cancer. There has been a large increase in the number of lung cancer cases in the last decade compared to the early 1990s [3]. Small cell lung cancer (SCLC) comprises approximately $12 \%$ of all lung cancers detected. SCLC is a highly malignant tumor of the lung and due to its rapid growth and development of drug resistance, its treatment still remains a tough task and challenge for scientists [4].

Chemotherapy is the main treatment strategy in both primary and palliative care of patients with lung cancer. Many patients with SCLC do not 
respond to such chemotherapy, or they respond well initially and then slowly decline. This may lead to an increase in the drug dosage, which generally increases the adverse effects, without improving the clinical outcome. In contrast to non-small cell lung cancer (NSCLC), small cell lung cancer cells (SCLC) can acquire resistance with prolonged use of the anticancer drugs [5]. Additionally, majority of the patients with lung cancer already have reached metastatic stage at the time of diagnosis making it difficult to use other treatment regimens like surgery and radiotherapy. If left untreated, SCLC has the most belligerent clinical course of any type of pulmonary tumor, with average survival from diagnosis of only 2 to 4 months [6].

Keeping in view the limited treatment options available for small cell lung cancer coupled with the menace of drug resistance which is rampant in most SCLC cancers, the objective of the present research work was to evaluate salvianolic acid, a natural product, for its anticancer effects against the human SCLC cell line $\mathrm{NCl}-\mathrm{H} 69$ (H69). We studied its effect on apoptosis induction, DNA damage and mitochondrial membrane potential loss in these cell lines using fluorescence microscopy, gel electrophoresis and flow cytometry. The compound exhibited dose-dependent as well as time-dependent antiproliferative effects against this multidrug resistant cell line. The results are encouraging since this cell line is highly resistant to most anticancer drugs and as such, salvianolic acid can be developed to target this cancer.

\section{EXPERIMENTAL}

\section{Cell lines and chemicals}

Human SCLC cell line NCl-H69 (H - 69) was procured from Shanghai Institute of Biochemistry and Cell Biology (Shanghai, China). Cells were cultured in RPMI 1640 with $10 \%$ fetal bovine serum (Hyclone Laboratories, Logan, UK) and $100 \mathrm{units} / \mathrm{ml}$ penicillin $-100 \mu \mathrm{g} / \mathrm{Ll}$ streptomycin, and were grown at $37{ }^{\circ} \mathrm{C}$ in a humidified $95 \%$ air and $5 \% \mathrm{CO}_{2}$ atmosphere. RPMI 1640 and other culture materials were from GIBCO/BRL Life Technologies (Grand Island, NY). Salvianolic acid A (> $95 \%$ purity by HPLC) was purchased from Sigma Chemical Co. (St. Louis, MO.).

\section{Cell proliferation assay using MTT}

Cell proliferation was determined by MTT assay. Human SCLC cells (H-69) in exponential growth were seeded at a final concentration of $5 \times 10^{5}$ cells $/ \mathrm{mL}$ in a 96 -well plate. At $50 \%$ confluence, the cells were pretreated with salvianolic acid $A$ at various concentrations $(0,5,10,20,40,80$ and 100) for $24 \mathrm{~h}$. Ten microliters of MTT solution (1 $\mathrm{mg} / \mathrm{mL}$ in PBS) was added to each well and the cells were cultured for another $4 \mathrm{~h}$ at $37^{\circ} \mathrm{C}$. The medium was completely removed and $200 \mu \mathrm{L}$ DMSO was added to solubilize MTT formazan crystals. The plates were then agitated and the optical density was determined at 570 $\mathrm{nm}$ (OD570) using an ELISA plate reader (Model 550; Bio-Rad, Hercules, CA, USA). At least three independent experiments were performed.

\section{Computer-assisted phase contrast microscopy (quantitative videomicroscopy)}

As described in previous studies [7,8], the salvianolic acid A-induced cytotoxic effects against the human SCLC cells $(H$ - 69) were directly visualized using quantitative videomicroscopy. This experiment involving computer-assisted phase contrast microscopy was developed to capture digital images of the cell culture at a different treatment concentrations $(0,10,50$ and $100 \mu \mathrm{M})$ for a period of $48 \mathrm{~h}$ thus providing 1200 high quality images that can be watched as running movies of few minutes length.

\section{Nuclear staining with Hoechst $\mathbf{3 3 2 5 8}$ for cell morphology study}

Human SCLC cells (H-69, $1 \times 10^{5}$ cells/dish) were plated in 6-cm dish and then treated with or without salvianolic acid A (concentrations used were $0,10,50$ and $100 \mu \mathrm{M})$. After $48 \mathrm{~h}$ incubation, the harvested cells were washed with PBS and fixed with $1 \%$ glutaraldehyde for 20 min. After this the cells were stained with Hoechst 33258 for $10-12$ min. The cells were again washed with PBS and then observed by fluorescence microscope (Olympus, Olympus Optical Co., LTD, Tokyo, Japan) using UV filter at X40 magnification.

\section{Flow cytometry analysis of the mitochondrial membrane potential $(\Delta \Psi \mathrm{m})$}

The effect of salvianolic acid A on mitochondrial membrane potential in human SCLC cells was detected by using rhodamine-123 (2 mM) fluorescent probe. This probe aids in measuring membrane polarization in live cell mitochondria. The SCLS cells $\left(5 \times 10^{6}\right.$ cells/dish) were treated with different concentrations of salvianolic acid $A$ $(0,10,50$ and $100 \mu \mathrm{M})$ for $48 \mathrm{~h}$ and mitochondrial membrane potential was measured by flow cytometry using BD FACSCalibur flow cytometer and Cell Quest software 3.0 (BectonDickinson). Rhodamine-123 (2 mM) was added 2 $\mathrm{h}$ before the termination of experiment. Cells 
were collected, washed in PBS, and incubated with propidium iodide $(20 \mu \mathrm{g} / \mathrm{mL})$ for $20 \mathrm{~min}$.

\section{Analysis of DNA fragmentation after salvianolic acid-A treatment}

The SCLC cells $\left(5 \times 10^{5}\right.$ cells/dish) were plated in $6-\mathrm{cm}$ dish and then subjected to treatment with different concentrations of salvianolic acid A. After drug treatment, the cells were washed with ice-cold PBS and resuspended in lysis buffer $(25$ $\mathrm{mM}$ Tris- $\mathrm{HCl}, \mathrm{pH} 7.4,5 \mathrm{mM}$ EDTA and $0.6 \%$ SDS) with $0.5 \mathrm{mg} / \mathrm{mL}$ RNase A for $20 \mathrm{~min}$ at 50 ${ }^{\circ} \mathrm{C}$. After this proteinase $\mathrm{K}$ was added and then the cells were incubated overnight. Separation of DNA was done using $2 \%$ agarose gel and the DNA was detected under UV light after staining with ethidium bromide (ETBR).

\section{Statistical analysis}

All data were derived from at least three independent experiments. The results are expressed as mean $\pm S D$ in all conditions. Differences between groups were analyzed using Student's t-test. $P<0.05$ was considered statistically significant.

\section{RESULTS}

Antiproliferative activity of salvianolic acid-A against SCLC cells

The antiproliferative activity of salvianolic acid-A is shown in Fig. 1. The compound exhibited dose-dependent as well as time-dependent antiproliferative effects against this multidrug resistant cell line. The results are encouraging since this cell line is highly resistant to most anticancer drugs and such salvianolic acid can be developed to target this cancer.

\section{Quantitative videomicrograph}

The limitation of the MTT assay is that it does not inform us whether a compound reduces the growth of cancer cells through cytostatic or cytotoxic effects or a cumulative effect of several of these effects. Quantitative videomicroscopy or computer-assisted phase contrast microscopy allows us to differentiate between these different kinds of growth inhibitory effects of salvianolic acid-A against SCLC cells. The results of this experiment are shown in Fig. 2 A-D. Cells that died appeared rounded and refringent under quantitative videomicroscopy analysis. The proportion of this cell type in SCLC cells was higher following treatment with salvianolic acid at higher doses (B, C and D represent 10, 50 and $100 \mu \mathrm{M}$ concentration of salvianolic acid-A) indicating higher cell death at higher concentration of salvianolic acid-A. The results of this experiment showed that salvianolic acid-A exerted cytostatic effects and not cytotoxic effects against these cells. Indeed, salvianolic acid-A induced marked vacuolization processes, which in turn, led to cell death.

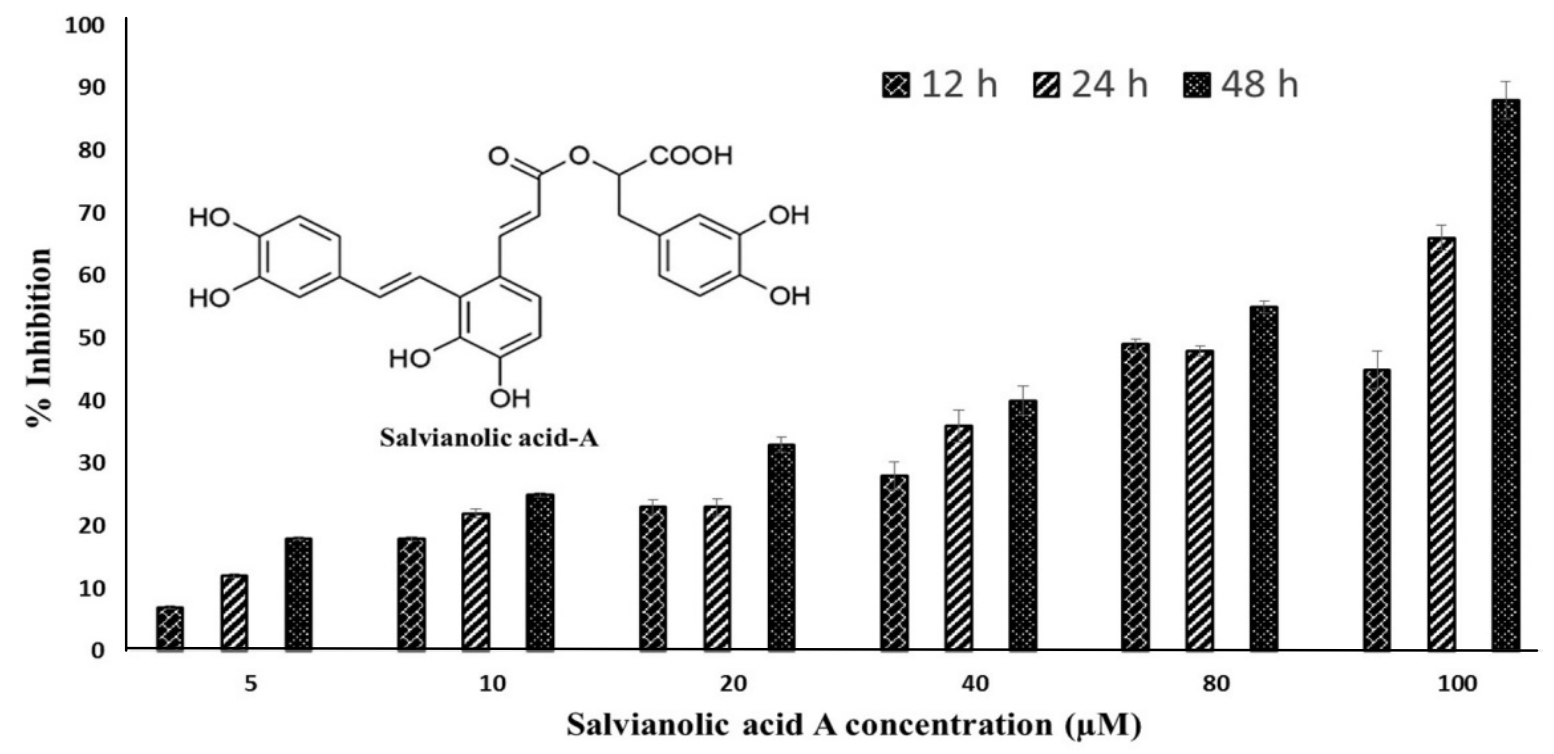

Figure 1: Antiproliferative effect of the different doses of salvianolic acid-A on the SCLC cells at three different time intervals (12 h, $24 \mathrm{~h}$ and $48 \mathrm{~h}$ ) 
Effect of salvianolic acid-A on apoptosis induction in SCLC cells

In order to evaluate whether the salvianolic acidA induced anticancer effects against SCLC cells was due to associated apoptosis, we evaluated the effect of salvianolic acid-A on nuclear morphology using Hoechst 33258 staining. Fig. 3 $A$, represents untreated cells which showed normal nuclear morphology, Fig. 3B-D represent 10,50 and $100 \mu \mathrm{M}$ doses of salvianolic acid-A respectively. The salvianolic acid-A treated cells showed dose dependent chromatin condensation which increases from A-D.
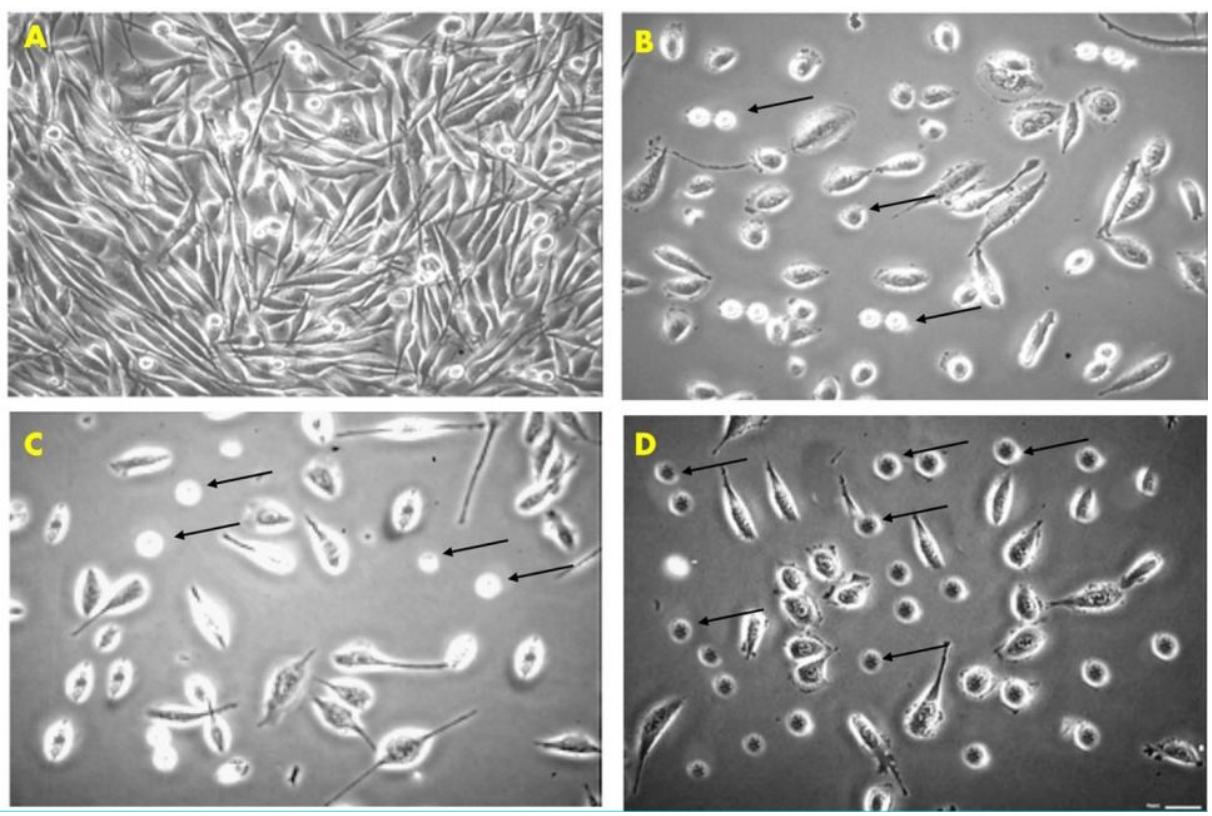

Figure 2: Quantitative videomicroscopy analysis salvianolic acid A for the human small cell lung cancer cancer cell line (H-69). The morphological analyses were carried out at a X100 magnification. The arrows show rounded cells which represent the dead cells. (A)represents untreated cells; (B) represents $10 \mu \mathrm{M},(\mathrm{C})$ represents $50 \mu \mathrm{M}$ and $(D)$ represents $100 \mu \mathrm{M}$ concentration of salvianolic acid-A. Data are expressed as the means \pm SEM
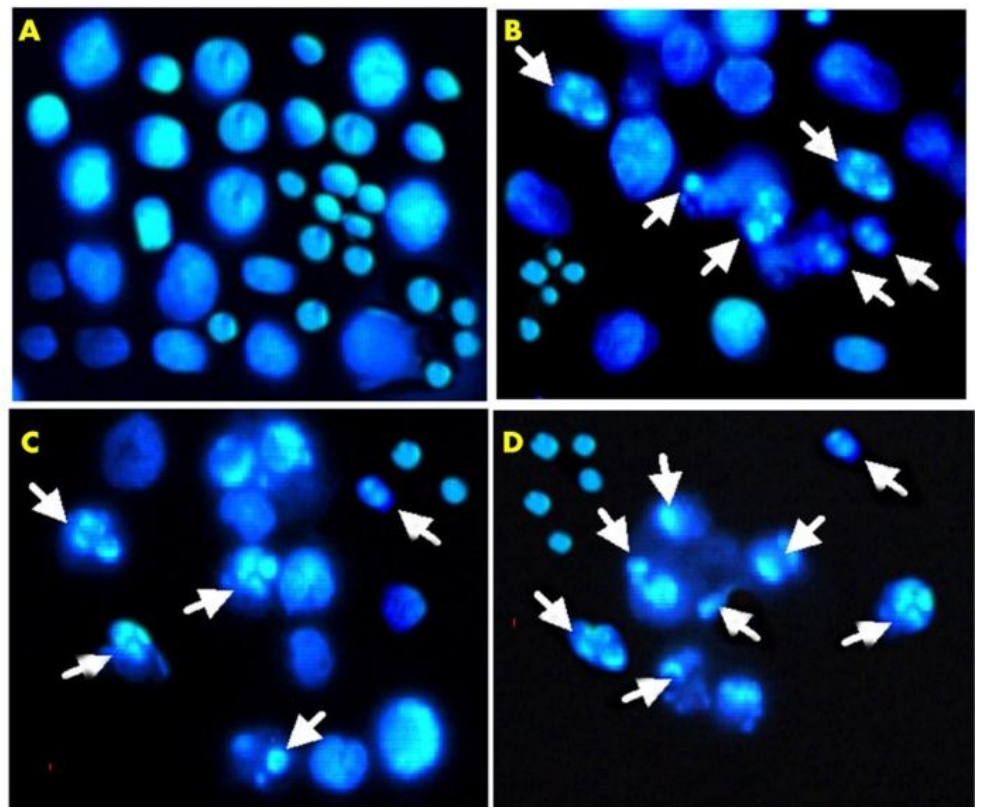

Figure 3: Effect of salvianolic acid-A on apoptosis induction (chromatin condensation) in human small cell lung cancer cells. SCLC cells were treated with $0 \mu \mathrm{M}(\mathrm{A}), 10 \mu \mathrm{m}(\mathrm{B}), 50 \mu \mathrm{m}(\mathrm{C})$ and $100 \mu \mathrm{m}(\mathrm{D})$ respectively for $48 \mathrm{~h}$ and stained with Hoechst 33258. The nuclear morphology was observed by fluorescent microscopy (magnification 400×). Arrows represent nuclear chromatin condensation 


\section{Effect of salvianolic acid-A on DNA fragmentation in SCLC cells}

The effects of the salvianolic acid-A on DNA fragmentation and hence on the apoptosis induction are shown in Fig. 4. DNA fragmentation was analyzed based on DNA laddering using agarose gel electrophoresis. Fig. 4 shows that salvianolic acid-A treatment led to the appearance of the DNA ladder in a dosedependent manner. $M$ is the 100-bp DNA marker. DNA fragmentation is a key event in the biochemical process of apoptosis.

\section{Salvianolic acid-A ( $\mathrm{MM})$}

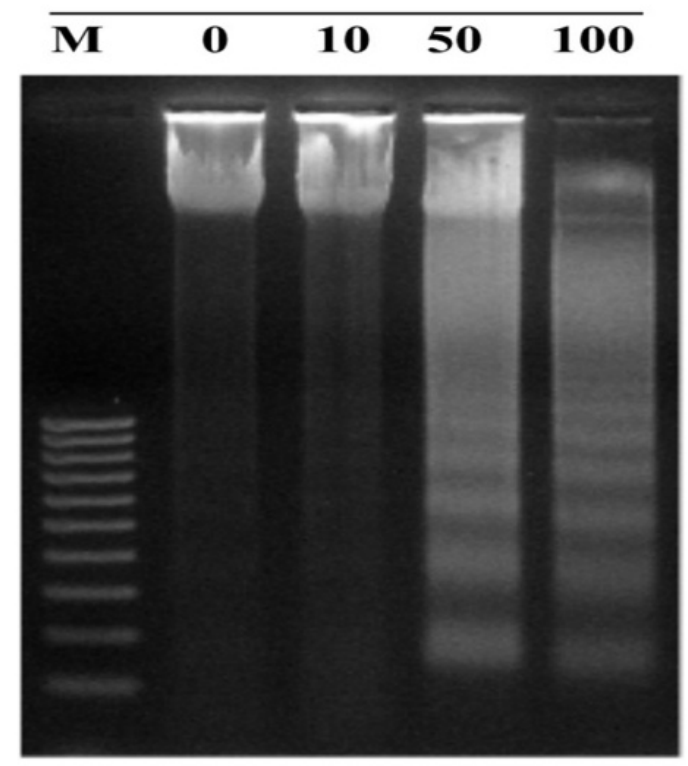

Figure 4: Induction of DNA fragmentation by salvianolic acid-A in human SCLC cells. The cells were treated with salvianolic acid at various concentrations $(0,10,50$ and $100 \mu \mathrm{M})$ for $48 \mathrm{~h}$, and the DNA fragmentation was analyzed by agarose gel electrophoresis. $M$ is the 100 -bp DNA marker

\section{Effect of salvianolic acid-A on the mitochondrial membrane potential in SCLC cells}

The results of the effect of salvianolic acid-A on mitochondrial membrane potential are shown in Fig. 5 A-D. As can be seen in Fig. 5, salvianolic acid induced concentration-dependent loss of mitochondrial membrane potential in SCLC cells. The number of cells with intact mitochondrial membrane potential decreased considerably while as the number of cells with decreased mitochondrial membrane potential increased. Fig. 5A represents untreated cells.

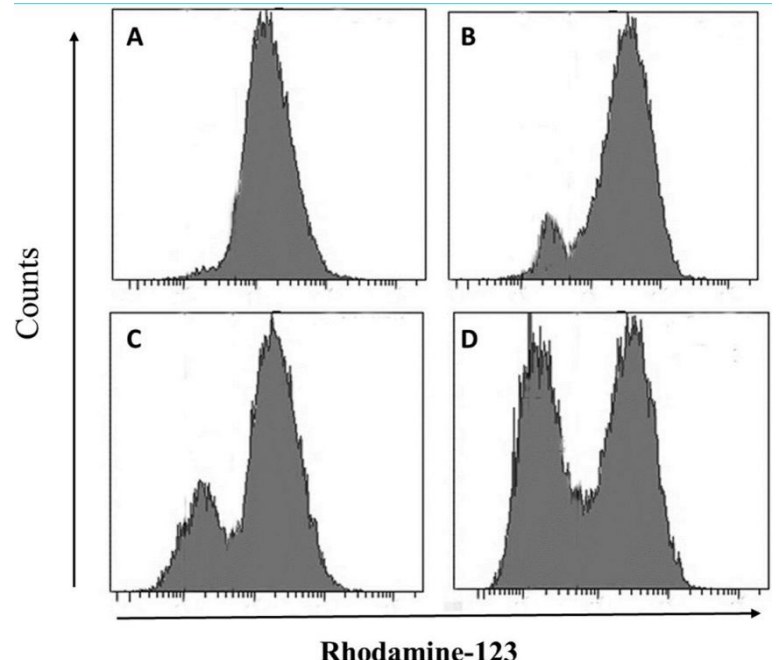

Figure 5: Salvianolic acid-A induced loss of mitochondrial membrane potential in SCLC cancer cells. A, represents untreated cells, B, C and D, represent 10,50 and $100 \mu \mathrm{M}$ concentration of salvianolic acid-A

\section{DISCUSSION}

Salvianolic acid $A$ is the most abundant, watersoluble and an effective compound extracted from Salvia miltiorrhiza (Danshen) which has been used in the treatment of various diseases. In China, Danshen has been wildly used to treat various diseases for hundreds of years. Salvianolic acids, especially salvianolic acid A and salvianolic acid $B$, have been found to have potent anti-oxidative capabilities due to their polyphenolic structure $[9,10]$. Salvianolic acid-A has been reported to exhibit anti-platelet aggregation and anticancer properties. It has also been reported that combination of salvianolic acid-A with other known anticancer agents like 5-fluorouracil, mitomycin $\mathrm{C}$ and methotrexate led to a potent cancer growth inhibition $[11,12]$. Preliminary data have indicated that salvianolic acid-A is able to inhibit cancer growth in mouse models, including liver, breast and lung cancers. The mechanism of action of salvianolic acid-A in the regulation of cancer cell growth has yet to be elucidated.

The objective of the current research work was to elucidate the molecular mechanism of action of salvianolic acid-A against small cell lung cancer cells. We studied the effect of salvianolic acid-A on apoptosis induction using computer-assisted phase contrast microscopy as well as fluorescence microscopy. These assays demonstrated how this compound affects nuclear chromatin condensation and overall cellular morphology. Gel electrophoresis indicated that salvianolic acid induced dose-dependent DNA 
damage in these cells. Flow cytometry analysis using rhodamine-123 fluorescent probe revealed that salvianolic acid induces potent loss in mitochondrial membrane potential which also followed dose-dependence.

\section{CONCLUSION}

This work reveals, probably for the first time, the molecular mechanism of salvianolic acid-A against small cell lung carcinoma. The compound induces potent growth inhibitory and apoptotic effects against this cell line. Therefore, salvianolic acid-A has the potential to be developed as an anticancer agent against this drug-resistant small cell lung carcinoma.

\section{REFERENCES}

1. Jemal A, Bray F, Center MM, Ferlay J, Ward E, Forman D. Global cancer statistics. CA Cancer J Clin 2011; 61(2): 69-90.

2. Siegel R, Ward E, Brawley O, Jemal A. Cancer statistics, 2011: the impact of eliminating socioeconomic and racial disparities on premature cancer deaths. CA Cancer J Clin 2011; 61: 212-236.

3. Chen W, Zhang S, Zou X. Estimation and projection of lung cancer incidence and mortality in China. Zhongguo Fei Ai Za Zhi 2010; 13(5): 488-493. (In Chinese).

4. Sethi T, Rintoul RC, Moore SM, MacKinnon AC, Salter D, Choo C, Chilvers ER, Dransfield I, Donnelly SC, Strieter $R$ et al. Extracellular matrix proteins protect small cell lung cancer cells against apoptosis: A mechanism for small cell lung cancer growth and drug resistance in vivo. Nat Med 1999; 5(6):662-668.
5. Manish S, David W, Jack A, Rajagopal R. Drug resistance in lung cancer. Lung Cancer: Targ Ther 2010; 2010(1):23-36.

6. Kavallaris $M$, Kuo DY, Burkhart $C A$, Regl DL, Norris MD, Haber M, Horwitz SB. Taxol-resistant epithelial ovarian tumors are associated with altered expression of specific beta-tubulin isotypes. J Clin Invest 1997; 100(5):1282-1293.

7. Mégalizzi V, Mathieu V, Mijatovic T, Gailly P, Debeir O, De Neve N, Van Damme M, Bontempi G, HaibeKains B, Decaestecker $C$ et al. 4-IBP, A sigma1 receptor agonist, Decreases the migration of human cancer cells, Including glioblastoma cells, in vitro and sensitizes them in vitro and in vivo to cytotoxic insults of proapoptotic and proautophagic drugs. Neoplasia 2007; 9(5): 358-369.

8. Delbrouck C, Doyen I, Belot N, Decaestecker C, Ghanooni $R$, de Lavareille A, Kaltner $H$, Choufani $G$, Danguy $A$, Vandenhoven $G$ et al. Galectin-1 is over expressed in nasalpolyps under budesonide and inhibits eosinophil migration. Lab Invest 2002; 82(2): 147-158.

9. Zhang $L$, Zhang $W$, Zhao $Y$, Yang $X$, Fang $L$, Wang $S$, Shi L, Yu $X$, Wang $S$, Yang $H$ et al. Research progress of salvianolic acid A. Abbreviatei 2011; 36:2603-2609. (In Chinese).

10. Zhou L, Zuo Z, Chow MS. Danshen: an overview of its chemistry, pharmacology, pharmacokinetics, and clinical use. J Clin Pharmacol 2005; 45(12):13451359.

11. Zhang W, Lu Y. Advances in studies on antitumor activities of compounds in Salvia miltiorrhiza. Zhongguo Zhong Yao Za Zhi 2010; 35(3):389-392. (In Chinese).

12. Zhang S, Su J, Zhen Y. Salvianolic acid $A$ inhibits nucleoside transport and potentiates the antitumor activity of chemotherapeutic drugs. Yao Xue Xue Bao 2004; 39(7):496-499. (In Chinese). 